\title{
Spin-phonon coupling effects in transition-metal perovskites: a DFT $+U$ and hybrid-functional study
}

\author{
Jiawang Hong, ${ }^{1, \text { * }}$ Alessandro Stroppa,${ }^{2}$ Jorge Íniguez, ${ }^{3}$ Silvia Picozzi, ${ }^{2}$ and David Vanderbilt ${ }^{1}$ \\ 1 Department of Physics and Astronomy, Rutgers University, Piscataway, NJ 08854-8019, USA \\ ${ }^{2}$ CNR-SPIN, L'Aquila, Italy \\ ${ }^{3}$ Institut de Ciència de Materials de Barcelona (ICMAB-CSIC), Campus UAB, 08193 Bellaterra, Spain
}

(Dated: September 20, 2018)

\begin{abstract}
Spin-phonon coupling effects, as reflected in phonon frequency shifts between ferromagnetic (FM) and G-type antiferromagnetic (AFM) configurations in cubic $\mathrm{CaMnO}_{3}, \mathrm{SrMnO}_{3}, \mathrm{BaMnO}_{3}, \mathrm{LaCrO}_{3}$, $\mathrm{LaFeO}_{3}$ and $\mathrm{La}_{2}(\mathrm{CrFe}) \mathrm{O}_{6}$, are investigated using density-functional methods. The calculations are carried out both with a hybrid-functional (HSE) approach and with a DFT $+U$ approach using a $U$ that has been fitted to HSE calculations. The phonon frequency shifts obtained in going from the FM to the AFM spin configuration agree well with those computed directly from the more accurate HSE approach, but are obtained with much less computational effort. We find that in the $A \mathrm{MnO}_{3}$ materials class with $A=\mathrm{Ca}, \mathrm{Sr}$, and $\mathrm{Ba}$, this frequency shift decreases as the A cation radius increases for the $\Gamma$ phonons, while it increases for R-point phonons. In $\mathrm{La} \mathrm{O}_{3}$ with $M=\mathrm{Cr}, \mathrm{Fe}$, and $\mathrm{Cr} / \mathrm{Fe}$, the phonon frequencies at $\Gamma$ decrease as the spin order changes from AFM to FM for $\mathrm{LaCrO}_{3}$ and $\mathrm{LaFeO}_{3}$, but they increase for the double perovskite $\mathrm{La}_{2}(\mathrm{CrFe}) \mathrm{O}_{6}$. We discuss these results and the prospects for bulk and superlattice forms of these materials to be useful as multiferroics.
\end{abstract}

PACS numbers: 75.85.+t, 75.47.Lx, 63.20.dk, 77.80.bg

\section{INTRODUCTION}

Multiferroic materials are compounds showing coexistence of two or more ferroic orders, e.g., ferroelectricity together with some form of magnetic order such as ferromagnetism or antiferromagnetism. Presently such materials are attracting enormous attention due to their potential for advanced device applications and because they offer a rich playground from a fundamental physics point of view. Possible applications tend to focus on the magnetoelectric (ME) coupling, which may pave the way for control of the magnetization by an applied electric field in spintronic devices,,$\stackrel{1,2}{,}$ although other applications such as four-state memories $\frac{3}{-1}$ are also of interest.

Although intrinsic multiferroic materials are highly desirable, they are generally scarce. One often-proposed reason may be that partially filled $3 d$ shells favor magnetism, while the best-known ferroelectric (FE) perovskites have a $3 d^{0}$ configuration for the transition metal (e.g. $\mathrm{Ti}^{4+}, \mathrm{Nb}^{5+}$, etc. $\stackrel{4}{ }$ ). However, it has recently been shown ${ }^{5}$ that some magnetic perovskite oxides display incipient or actual FE instabilities, clearly indicating that there are ways around the usually-invoked incompatibility between ferroelectricity and magnetism. Some of these compounds, namely $\mathrm{CaMnO}_{3}, \mathrm{SrMnO}_{3}$ and $\mathrm{BaMnO}_{3}$, will be considered in this work.

Even if several microscopic mechanisms have been identified for the occurrence of ferroelectricity in magnetic materials,,$\frac{6,7}{7}$ alternative routes are needed in order to optimize materials for functional device application. This could be done either by focusing on new classes of compounds such as hybrid organic-inorganic materials ${ }^{8-10}$ or by modifying already-known materials to engineer specific properties. In the latter direction, an intriguing possibility is to start with non-polar ma- terials and then induce multiple non-polar instabilities; under appropriate circumstances this can produce a ferroelectric polarization, as first predicted in Ref. 11 based on general group theory arguments and analyzed in the $\mathrm{SrBi}_{2} \mathrm{Nb}_{2} \mathrm{O}_{9}$ compound by means of a symmetry analysis combined with density-functional theory calculations by Perez-Mato et al $\stackrel{12}{ }$ Here the ferroelectricity was found to arise from the interplay of several degrees of freedom, not all of them associated with unstable or nearly-unstable modes. In particular, a coupling between polarization and two octahedral-rotation modes was invoked to explain the behavior $\stackrel{12}{\underline{1}}$ Bousquet et al. have demonstrated that ferroelectricity is produced by local rotational modes in a $\mathrm{SrTiO}_{3} / \mathrm{PbTiO}_{3}$ superlattice ${ }^{13}$ Although in most improper ferroelectrics a single primary order parame-

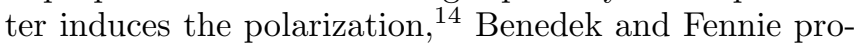
posed that the combination of two lattice rotations, neither of which produces ferroelectric properties individually, can induce a ME coupling, weak ferromagnetism, and ferroelectricity 15 Indeed, we now know that rotations of the oxygen octahedra, in combination $\frac{15-17}{17}$ and even individually, $\underset{18,19}{19}$ can produce ferroelectricity, modify the magnetic order, and favor magnetoelectricity.

Another route to creating new multiferroic materials may be to exploit the coupling between polarization, strain, and spin degrees of freedom. A strong dependence of the lowest-frequency polar phonon frequency on epitaxial strain 20 is common in paraelectric $(\mathrm{PE})$ perovskite oxides, and can sometimes be exploited to drive the system ferroelectric, a phenomenon known as epitaxialstrain-induced ferroelectricity. ${ }^{21}$ In a magnetic system that also has a strong spin-phonon coupling, i.e., a strong dependence of the polar phonon frequencies on spin order, the magnetic order may be capable of tipping the balance between PE and FE states. For example, con- 
sider a system that has two competing ground states, one of which is antiferromagnetic (AFM) and PE while the other is ferromagnetic (FM) and FE, and assume that the spin-phonon coupling is such that the lowest-frequency polar phonon is softer for FM ordering than for AFM ordering. In such a case, the epitaxial-strain enhancement of a polar instability may lead to a lowering of the energy of the FM-FE state below that of the AFM-PE phase. Spin-phonon mechanisms of this kind have been powerfully exploited for the design of novel multiferroics in Refs. 22 28.

Clearly, it is desirable to have a large spin-phonon coupling, in terms of a large shift $\Delta \omega$ of phonon frequencies upon change of the magnetic order. Interestingly, recently computed spin-phonon couplings in ME compounds seems to be strikingly large. For example, a $\Delta \omega$ of about $60 \mathrm{~cm}^{-1}$ has been reported for the double perovskite $\mathrm{La}_{2} \mathrm{NiMnO}_{6} \cdot \frac{29}{}$ Furthermore, $\Delta \omega$ values of about $200 \mathrm{~cm}^{-1}$ have been computed for a cubic phase of $\mathrm{SrMnO}_{3} \underline{\underline{30}}$ These values appear to be anomalously large when compared to phonon splittings across magnetic phase transitions in other oxides, which are in the $5-30 \mathrm{~cm}^{-1}$ range $\underline{31.32}$

In the search for materials with large spin-phonon coupling, first-principles based calculations play a prominent role since they can pinpoint promising candidates simply by inspecting the dependence of the phonon frequencies on the magnetic order. However, a serious bottleneck appears. To obtain a reliable and accurate description of these effects, it is important to describe the structural, electronic and magnetic properties on an equal footing. This is especially true for transition-metal oxides, which are the usual target materials for the spinphonon driven ferroelectric-ferromagnet. In this case, it is well known that the localized nature of the $3 d$ electronic states, or loosely speaking, the "correlated" nature of these compounds, poses serious limits to the applicability of common density-functional methods like the local density approximation (LDA) or generalized gradient approximation (GGA). In fact, these standard approximations introduce a spurious Coulomb interaction of the electron with its own charge, i.e., the electrostatic self-interaction is not entirely compensated. This causes fairly large errors for localized states (e.g., Mn $d$ states). It tends to destabilize the orbitals and decreases their binding energy, leading to an overdelocalization of the charge density $\underline{33}$

One common way out is the use of the DFT $+U$ method,, 34 where a Hubbard-like $U$ term is introduced into the DFT energy functional in order to take correlations partially into account. The method usually improves the electronic-structure description, but it suffers from shortcomings associated with the $U$-dependence of the calculated properties ${ }^{35}$ Unfortunately, there is usually no obvious choice of the $U$ value to be adopted; common choices are usually based either on experimental input or are derived from constrained DFT calculations, but neither of these approaches is entirely satis- factory. When dealing with phonon calculations, the $U$ dependence becomes even more critical since the phonon frequencies depend strongly on the unit-cell volume used for their evaluation, and the theoretical volume, in turn, depends on $U$. It is worth mentioning that even if an appropriate choice of $U$ can accurately reproduce the binding energy of localized $d$ states of transition-metal oxides, it is by no means guaranteed that the same $U$ can accurately reproduce other properties of the same compound, such as the volume $\stackrel{35,36}{ }$ While most papers address the spin-phonon coupling by applying DFT $+U$ methods, only a few deal with the dependence upon the $U$ parameter ${ }^{37}$ In this paper, we will show that the spinphonon coupling can strongly depend on the $U$ parameter, and that such a dependence may give rise to artificially large couplings. It is important to stress this message, often overlooked in the literature, in view of the increasing interest in ab-initio predictions of ferroelectric materials driven by spin-phonon coupling.

In the last few years, another paradigmatic approach has been widely applied in solid-state materials science, namely the use of "hybrid functionals" that incorporate a weighted mixture of the exchange defined in the HartreeFock theory (but using the Kohn-Sham orbitals) and the density-functional exchange. The correlation term is retained from the density-functional framework. It is now widely accepted that the hybrid functionals outperform semilocal functionals, especially for bulk materials with band gaps $\frac{38-49}{4}$ It has also been shown that for lowdimensional systems such as semiconductor/oxides interfaces, the performance of hybrid functionals remains quite satisfactory $\underline{\underline{50}}$ However, some doubts have very recently been put forward about the performance of hybrid functionals for certain structural configurations, e.g., surfaces or nanostructures $\underline{51}$

While there is a plethora of different hybrid functionals, many of them defined empirically, a suitable functional derived on theoretical grounds is the so called PBE0,$\underline{52.53}$ where the exchange mixing parameter as been fixed to one quarter as justified by a perturbation-theory calculation. A closely related functional, the HeydScuseria-Ernzerhof (HSE) hybrid functional, $\underline{\underline{54}}$ introduces yet another parameter $\mu$ which splits the Coulomb interaction kernel into short- and long-range pieces while retaining the mixing only on the short-range component. It has been shown that this new hybrid functional, 54 while preserving most of the improved performance of PBE0 with respect to standard local and semi-local exchange correlation functionals, greatly reduces the computational cost. For this reason, it is especially suitable for periodically extended systems, and is currently being applied in many solid-state applications ranging from simple semiconductor systems to transition metals, lanthanides, actinides, molecules at surfaces, diluted magnetic semiconductors, and carbon nanostructures (for a recent review see Ref. 55). The HSE functional has been also used for phonon calculations for simple semiconducting systems $\frac{56,57}{5}$ or perovskite structures,$\underline{58,59}$ where it 
was shown the that phonon modes are much more accurately reproduced using the hybrid functionals than using GGA or LDA. 60

Very recently, extended benchmarkings of the HSE method as well as other self-interaction-corrected approaches have been presented for prototypical transition metal oxides such as $\mathrm{MnO}, \mathrm{NiO}$ and $\mathrm{LaMnO}_{3}$, and it has been shown that "HSE shows a remarkable quantitative agreement with experiments on most examined properties" 61 and "HSE shows predictive power in describing exchange interactions in transition metal oxides." 62 These recent studies further motivate us to use the HSE functional for our studies of the spin-phonon coupling; indeed, as we will see in Sec. IIIB the HSEcalculated phonon modes agree well with available experimental results for $\mathrm{SrMnO}_{3}$. The spin-phonon coupling effect, to the best of our knowledge, is totally unexplored by hybrid functional approaches. In this work we aim at filling this gap.

Even when using the more efficient HSE functional, however, the use of hybrids entails an increased computational cost which makes the calculation of phonon properties of complex magnetic oxides very difficult. In this paper, we propose to circumvent this bottleneck by combining the HSE and DFT $+U$ approaches, i.e., choosing the appropriate $U$ for each material by fitting to the HSE results for some appropriate materials properties. This provides a fairly efficient and affordable strategy that preserves the "HSE accuracy" for lattice constants, spinphonon couplings, and related properties, while taking advantage of the computationally inexpensive $\mathrm{DFT}+U$ method for the detailed calculations. Further details will be given in Section II.

As far as the materials are concerned, it has been suggested that $A \mathrm{MnO}_{3}$ perovskites with $A=\mathrm{Ca}, \mathrm{Sr}$ and Ba may be good candidates for spin-phonon-coupling driven multiferroicity. ${ }^{5,30,63}$ Furthermore, it has been reported that both $\mathrm{SrMnO}_{3}$ and $\mathrm{CaMnO}_{3}$ have a large spin-phonon coupling. ${ }^{30,64}$ We will show below that the spin-phonon coupling can depend strongly on the chosen $U$. We will also consider the simple perovskite $\mathrm{LaMO}_{3}$ materials with $M=\mathrm{Cr}$ and Fe, which have Néel temperatures above room temperature ${ }^{65}$ and large band gaps. We want to explore the possibility of using these two materials as building blocks for room temperature multiferroics, e.g., in the form of double perovskites such as $\mathrm{La}_{2} \mathrm{CrFeO}_{6}$. We have chosen these two classes of materials in part for the reasons outlined above, but also because they are sufficiently "easy-to-calculate" for the benchmark and testing purposes of the present work, especially when considering hybrid functionals.

The paper is organized as follows. In Sec. II we report the computational details and describe our strategy for fitting $U$ of the GGA $+U$ calculations via a preliminary "exploratory" study using the HSE method. In Sec. III we discuss: i) the effect of $U$ on the frequency shift, in Sec.III A ii) the spin-phonon coupling effects in $A \mathrm{MnO}_{3}$ with $A=\mathrm{Ca}, \mathrm{Sr}$, and $\mathrm{Ba}$, in Sec. IIIB, iii) the spin-phonon coupling effects in $\mathrm{La} \mathrm{O}_{3}$ with $M=\mathrm{Cr}, \mathrm{Fe}$, and the corresponding double perovskite $\mathrm{La}(\mathrm{Cr}, \mathrm{Fe}) \mathrm{O}_{3}$ in Sec. IIIC and iv) prospects for the design of new multiferroics in Sec. IIID, In Sec. IV] we give a summary and conclusions. Finally, in the Appendix, we give details about the methodology used here for a full HSE phonon calculation.

\section{METHODS}

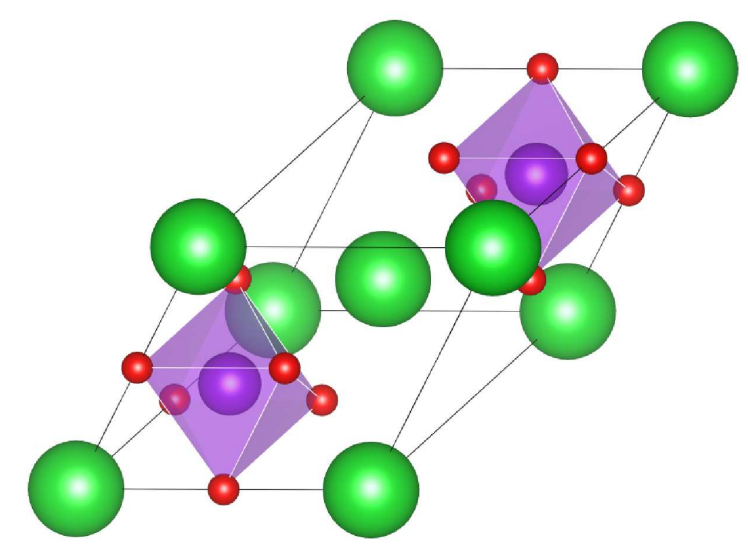

FIG. 1: (Color online) $\mathrm{ABO}_{3}$ perovskite structure doubled along the [111] direction. $A$ atoms (largest) shown in green; $B$ atoms shown in violet; $\mathrm{O}$ atoms (smallest) shown in red.

All of our calculations were performed in the framework of density-functional theory as implemented in the Vienna ab-initio simulation package (VASP-5.2) with a plane-wave cutoff of $500 \mathrm{eV}$. The $\mathrm{DFT}+U$ and HSE calculations were carried out using the same set of projector-augmented-wave (PAW) potentials to describe the electron-ion interaction ${ }^{68,69}$ The unit cell for simulating the G-AFM magnetic ordering, where all firstneighbor spins are antiferromagnetically aligned, is doubled along the [111] direction as shown in Fig. 1]. The same simulation cell is retained for the FM configuration in order to avoid numerical artefacts that could arise in comparing calculations with different effective k-point samplings. A $6 \times 6 \times 6 \Gamma$-centered $k$-point mesh is used.

The phonon frequencies were calculated using the frozen-phonon method. Except for the case of $\mathrm{La}_{2} \mathrm{CrFeO}_{6}$, the charge density and dynamical matrix retain the primitive 5-atom-cell periodicity for both the GAFM and FM spin structures, so it is appropriate to analyze the phonons using symmetry labels from the primitive $\operatorname{P} m \overline{3} m$ perovskite cell. The zone-center phonons decompose as $3 \Gamma_{4}^{-} \oplus \Gamma_{5}^{-}$(plus acoustic modes), with the $\Gamma_{4}^{-}$modes being polar. The zone-boundary modes at the $\mathrm{R}$ point (which appear at the $\Gamma$ point in our 10-atom-cell calculations) decompose as $\mathrm{R}_{5}^{+} \oplus \mathrm{R}_{5}^{-} \oplus 2 \mathrm{R}_{4}^{-} \oplus \mathrm{R}_{2}^{-} \oplus \mathrm{R}_{3}^{-}$, 

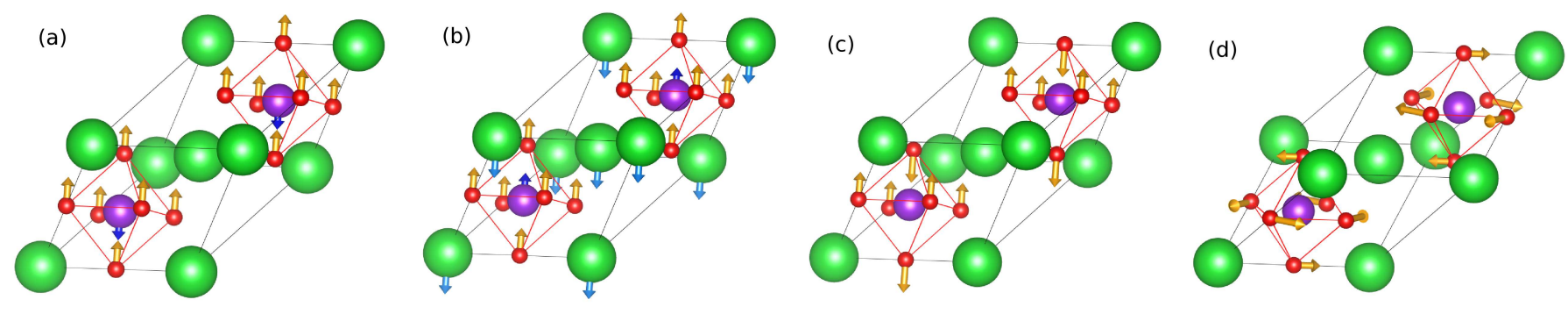

FIG. 2: (Color online) Three idealized polar modes and $R_{5}^{-}$mode in simple perovskites. (a) Slater mode; (b) Last mode; (c) Axe mode; (d) $R_{5}^{-}$mode.

where the three-fold degenerate $\mathrm{R}_{5}^{-}$is of special interest because it corresponds to rigid rotations and tilts of the oxygen octahedra. After each frozen-phonon calculation of the zone-center phonons of our 10-atom cell, we analyze the modes to assign them according to these $k$ point and symmetry labels. For $\mathrm{La}_{2} \mathrm{CrFeO}_{6}$, some of the modes mix (e.g., $\Gamma_{4}^{-}$with $\mathrm{R}_{5}^{+}$); in these cases we report the dominant mode character.

As for the polar $\Gamma_{4}^{-}$phonons, these are often characterized in $\mathrm{ABO}_{3}$ perovskites in terms of three kinds of idealized modes as illustrated in Figs. $2 \mathrm{a}$-c. The Slater mode $\left(\mathrm{S}-\Gamma_{4}^{-}\right)$of Fig. 2(a) describes the vibration of $B$ cations against the oxygen octahedra; the Last (L$\Gamma_{4}^{-}$) mode of Fig. 2(b) expresses a vibration of the $A$ cations against the $\mathrm{BO}_{6}$ octahedra; and the Axe mode $\left(\mathrm{A}-\Gamma_{4}^{-}\right)$of Fig. 2(c) represents the distortion of the oxygen octahedra $\stackrel{70}{ }$ The actual mode eigenvectors never behave precisely like these idealized cases, but we find that they can be identified in practice as being mainly of one character, which is the one we report. These polar modes contribute to the low-frequency dielectric constant, and their softening in the high-symmetry paraelectric phase indicates the presence of a ferroelectric instability. For the insulating compounds, we further calculated their dielectric constants and Born effective charges using density-functional perturbation theory within the $\mathrm{DFT}+U$ context as implemented in VASP. The antiferrodistortive (AFD) mode $\left(R_{5}^{-}\right.$mode), which describes the rotation oxygen octahedra, is also shown in Fig. 2(d).

We make use of the $\mathrm{DFT}+U \operatorname{method}^{34}$ in the standard Dudarev implementation where the on-site Coulomb interaction for the localized $3 d$ orbitals is parametrized by $U_{\text {eff }}=U-J$ (which we denote henceforth as simply $\left.U\right)^{71}$ using the PBEsol functional,,$\frac{72}{2}$ which has been shown to give a satisfactory description of solid-state equilibrium properties. We shall refer to this as PBEsol $+U$. The lack of experimentally available data for our systems prevents us from extracting $U$ directly from experiments; we will return to this delicate point shortly.

The other functional we have used is HSE06,,$\frac{54}{a}$ a screened hybrid functional introduced by Heyd, Scuseria, and Ernzerhof (HSE), where one quarter of the PBE short-range exchange is replaced by exact exchange, while the full PBE correlation energy is included. The range-separation parameter $\mu$ is set to $\mu=0.207 \AA^{-1}$. The splitting of the Coulomb interaction into short- and long-range pieces, as done in HSE, allows for a faster numerical convergence with $k$-points when dealing with solid-state systems. However, as previously mentioned, the application of the HSE approach to phonon calculations for magnetic oxide systems remains very challenging in terms of computational workload.

\section{Scheme to fit $U$ from hybrid calculations}

Here, we propose a practical scheme to perform relatively inexpensive $\mathrm{DFT}+U$ simulations that retain an accuracy comparable to HSE for the calculation of spinphonon couplings.

Our goal is to obtain a DFT $+U$ approach that reproduces the dependence of the materials properties on the spin arrangement as obtained from HSE calculations. Naturally, the first and most basic property we would like to capture correctly is the energy itself. As it turns out, the energy is also a very sensible property on which one can base a $U$-fitting scheme: The energy differences between spin configurations are directly related to the magnetic interactions or exchange constants, and these are known to depend on the on-site Coulomb repulsion $U$ affecting the electrons of the magnetic species. (Typically, in our compounds of interest, the value of $U$ used in the simulations will play an important role in determining the character of the top valence states; in turn, this will have a direct impact on the nature and magnitude of the exchange couplings between spins.) This dependence of the exchange constants on $U$ makes this criterion a very convenient one for our purposes. Of course, such a fitting procedure does not guarantee our DFT $+U$ scheme will reproduce correctly the phonon frequencies and frequency shifts between different spin arrangements obtained from HSE calculations. In that sense, we are relying on the physical soundness of the Hubbard- $U$ correction to DFT; as we will see below, the results are quite convincing.

Obtaining $U$ from the energy differences has an additional advantage: It allows us to devise a very simple fitting procedure that relies on a minimal number of HSE 


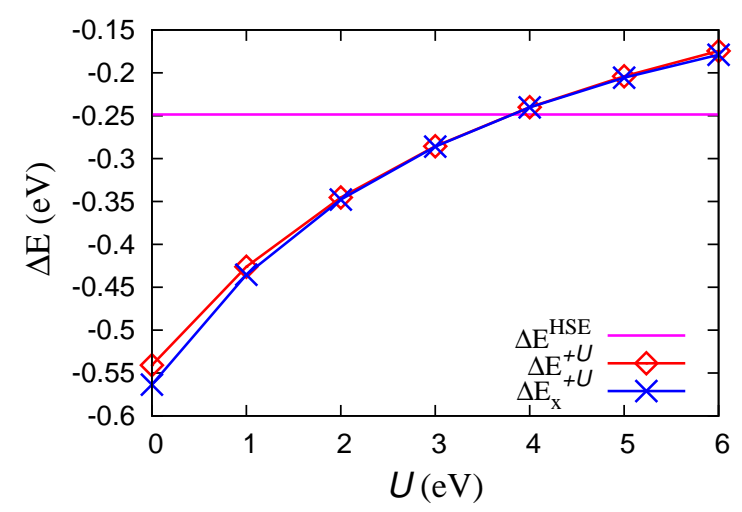

FIG. 3: (Color online) Variation of PBEsol $+U$ total energy difference $\Delta E=E_{\mathrm{AFM}}-E_{\mathrm{FM}}$ with $U$ for $\mathrm{LaCrO}_{3}, \Delta E^{+U}$ is the energy difference with volume fixed to the optimized volume from HSE and $\Delta E_{x}^{+U}$ relaxed volume for each $U$. (The fitted value of $U$ occurs at the crossing with the $\Delta E^{\mathrm{HSE}}$ value.)

calculations. In essence, these are the steps we follow. We relax the cubic structure separately for G-AFM and FM spin configurations using HSE and obtain the totalenergy difference $\Delta E$ (HSE) $=E_{\mathrm{AFM}}$ (HSE) - $E_{\mathrm{FM}}(\mathrm{HSE})$. We then carry out a series of PBEsol $+U$ calculations in which $U$ is varied from 0 to $6 \mathrm{eV}$ and obtain $\Delta E(+U)=$ $E_{\mathrm{AFM}}(+U)-E_{\mathrm{FM}}(+U)$ for each $U$. The $U$ is then chose such that $\Delta E(+U)=\Delta E(\mathrm{HSE})$.

Figure 3 shows the results of our fitting for $\mathrm{LaCrO}_{3}$ by using two slightly different approaches. In one case we ran the PBEsol $+U$ simulations at the HSE-optimized volumes, and in the other case we performed volume relaxations at the PBEsol+ $U$ level for each $U$ value considered. It can be seen that such a choice does not have a big effect on the computed frequency shifts, and both result in the same $U$ value. Hence, the $U$ values reported here were obtained by running PBEsol $+U$ simulations at the HSE volumes $\stackrel{73}{ }$ We obtained $U=3.0,2.8,2.7,3.8$, and $5.1 \mathrm{eV}$, respectively, for $\mathrm{CaMnO}_{3}, \mathrm{SrMnO}_{3}, \mathrm{BaMnO}_{3}$, $\mathrm{LaCrO}_{3}$ and $\mathrm{LaFeO}_{3} \stackrel{\underline{74}}{\underline{ }}$ We then do detailed phonon calculations using this value of $U$ in the PBEsol $+U$ calculations to investigate spin-phonon coupling effects in $A \mathrm{MnO}_{3}$ and $\mathrm{LaMO}_{3}$.

As we will show in Sec. III, we have tested this proposed scheme and found that it works quite well. In particular, the phonon frequency shifts $\Delta \omega=\omega_{\mathrm{AFM}}-\omega_{\mathrm{FM}}$ computed using PBEsol $+U$ with the fitted $U$ are almost the same as those obtained using HSE directly. Since a previous study has concluded that HSE works "remarkably well" for transition-metal oxides, $\frac{61}{\underline{n}}$ we believe this approach can be used with confidence.

Finally, we note that the direct HSE calculations can be rather heavy, even though we only have 10-atom cells; the presence of magnetic order and the need to calculate the phonons and the spin-phonon couplings makes the calculations challenging. $\underline{\underline{75}}$ We circumvent this difficulty
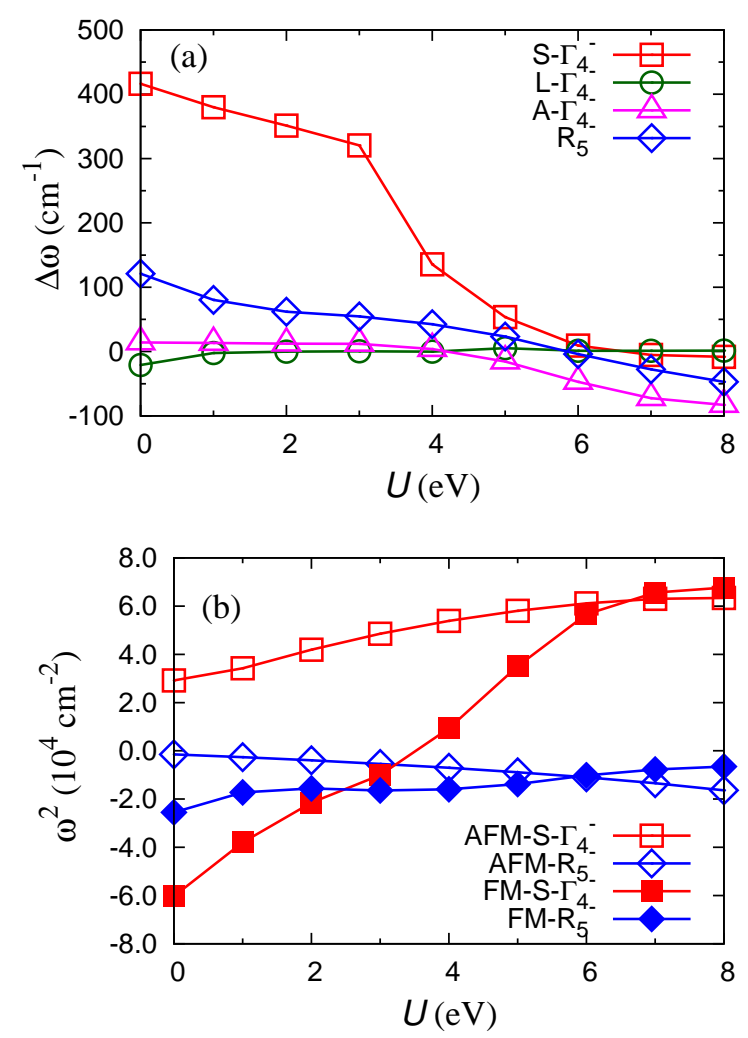

FIG. 4: (Color online) Effect of $U$ on spin-phonon coupling in $\mathrm{SrMnO}_{3}$. (a) $\Gamma_{4}^{-}$and $\mathrm{R}_{5}^{-}$phonon frequency shifts $(\Delta \omega=$ $\left.\omega_{\mathrm{AFM}}-\omega_{\mathrm{FM}}\right)$ versus $U$. (b) $\mathrm{S}-\Gamma_{4}^{-}$and $\mathrm{R}_{5}^{-}$phonon frequencies versus $U$ for AFM and FM states.

by splitting a single run of HSE frozen-phonon calculations into several parallel runs, each one computing the forces for symmetry-independent atomic displacements. We then use the forces calculated in these runs to construct the force-constant matrix, and by diagonalizing this matrix, we obtain the phonon frequencies and eigenvectors. Further details of this procedure are presented in the Appendix.

\section{RESULTS AND DISCUSSION}

\section{A. Effect of $U$ on the frequency shifts}

Let us begin by showing some representative results of the $U$-dependence of the phonon frequencies and frequency shifts for the $A \mathrm{MnO}_{3}$ and $\mathrm{LaMO}_{3}$ compounds. Here we focus on the case of $\mathrm{SrMnO}_{3}$, which has been predicted to exhibit a large spin-phonon coupling based on GGA $+U$ simulations using $U=1.7 \mathrm{eV}$; more precisely, a giant frequency shift $\Delta \omega=230 \mathrm{~cm}^{-1}$ has been reported for the Slater mode at the theoretical equilibrium state ${ }^{30}$

We computed the phonon frequencies and frequency shifts of the ideal cubic perovskite phase of $\mathrm{SrMnO}_{3}$ using $U$ values in the range between 0 and $8 \mathrm{eV}$. Figure 4 
TABLE I: Lattice constant $a$, local Mn magnetic moment $m$, and band gap $E_{\text {gap }}$ (zero if blank) for $\mathrm{CaMnO}_{3}, \mathrm{SrMnO}_{3}$ and $\mathrm{BaMnO}_{3}$ from PBEsol $+U(+U)$ and HSE methods.

\begin{tabular}{lcccc}
\hline \hline & & $a(\AA)$ & $m\left(\mu_{\mathrm{B}}\right)$ & $E_{\text {gap }}(\mathrm{eV})$ \\
\hline \multirow{2}{*}{$\mathrm{CaMnO}_{3}-\mathrm{AFM}$} & $+U$ & 3.73 & 2.79 & 0.35 \\
& $\mathrm{HSE}$ & 3.73 & 2.81 & 1.70 \\
$\mathrm{CaMnO}_{3}-\mathrm{FM}$ & $+U$ & 3.74 & 2.84 & \\
& $\mathrm{HSE}$ & 3.73 & 2.81 & \\
\hline \multirow{2}{*}{$\mathrm{SrMnO}_{3}-\mathrm{AFM}$} & $+U$ & 3.80 & 2.80 & 0.40 \\
& $\mathrm{HSE}$ & 3.80 & 2.85 & 1.45 \\
$\mathrm{SrMnO}_{3}-\mathrm{FM}$ & $+U$ & 3.81 & 2.89 & \\
& $\mathrm{HSE}$ & 3.81 & 2.91 & $0.00^{a}$ \\
\hline \multirow{2}{*}{$\mathrm{BaMnO}_{3}-\mathrm{AFM}$} & $+U$ & 3.91 & 2.86 & 0.13 \\
& $\mathrm{HSE}$ & 3.91 & 2.92 & 1.20 \\
$\mathrm{BaMnO}_{3}-\mathrm{FM}$ & $+U$ & 3.93 & 3.14 & \\
& $\mathrm{HSE}$ & 3.93 & 3.13 & \\
\hline \hline
\end{tabular}

${ }^{a}$ Half-metallic.

shows our results for the key modes that determine the occurrence of ferroelectricity, i.e., the $\Gamma_{4}^{-}$polar phonons and the $\mathrm{R}_{5}^{-}$antiferrodistortive (AFD) mode. The $\mathrm{R}_{5}^{-}$ mode involves antiphase rotations of the $\mathrm{O}_{6}$ octahedra around the three principal axes of the perovskite lattice; such a mode is soft in many cubic perovskites, and it often competes with the FE instabilities to determine the nature of the low-symmetry phases.

From Fig. 4 a we see that the $\Gamma_{4}^{-}$and $\mathrm{R}_{5}^{-}$modes depend strongly on the chosen $U$. Notably, the frequency shift $\Delta \omega$ of the $\mathrm{S}-\Gamma_{4}^{-}$mode can change from $400 \mathrm{~cm}^{-1}$ to $0 \mathrm{~cm}^{-1}$ as $U$ increases from 0 to $8 \mathrm{eV}$. The $\Delta \omega$ of the $\mathrm{A}-\Gamma_{4}^{-}$and $\mathrm{R}_{5}^{-}$modes also depend on the $U$, while the $\mathrm{L}-\Gamma_{4}^{-}$mode is nearly insensitive to $U$ within this range of values. Interestingly, the large frequency shift of $\mathrm{S}-\Gamma_{4}^{-}$ is related to the strong $U$-dependence of the $\mathrm{FM} \mathrm{S}-\Gamma_{4}^{-}$ mode, as shown in Fig. 4b.

Hence, our results show that the magnitude of the spin-phonon coupling has a significant dependence on the value of $U$ employed in a $\mathrm{DFT}+U$ calculation, and that such a dependence is particularly strong for some of the key modes determining the structural (FE/AFD) instabilities of cubic perovskite oxides. It is thus clear that choosing an appropriate $U$ is of critical importance if we want to avoid artificially "strong" couplings.

\section{B. Spin-phonon coupling in $\mathrm{CaMnO}_{3}, \mathrm{SrMnO}_{3}$, and $\mathrm{BaMnO}_{3}$}

We studied the cubic phase of $\mathrm{CaMnO}_{3}, \mathrm{SrMnO}_{3}$, and $\mathrm{BaMnO}_{3}$ using the PBEsol $+U$ approximation with $U$ values for Mn's $3 d$ electrons (i.e., $U=3.0 \mathrm{eV}$ for $\mathrm{CaMnO}_{3}, U=2.8 \mathrm{eV}$ for $\mathrm{SrMnO}_{3}$, and $U=2.7 \mathrm{eV}$ for $\mathrm{BaMnO}_{3}$ ) that were determined as described in Section III. The basic properties that we obtained are listed in Table II Our PBEsol $+U$ method gives good lattice
TABLE II: Phonon frequencies and frequency shifts calculated for $\mathrm{CaMnO}_{3}$, in $\mathrm{cm}^{-1}$. Frequencies calculated using HSE and PBEsol $+U: \omega^{\mathrm{HSE}}$ and $\omega^{+U}$. Frequency difference between HSE and PBEsol $+U: \omega^{\Delta}=\omega^{+U}-\omega^{\text {HSE }}$. Frequency shift: $\Delta \omega=\omega_{\mathrm{AFM}}-\omega_{\mathrm{FM}}$. Difference of frequency shifts between HSE and PBEsol $+U: \Delta \omega^{\Delta}=\Delta \omega^{+U}-\Delta \omega^{\mathrm{HSE}}$.

\begin{tabular}{lrrrrrr}
\hline \hline & $\omega_{\text {AFM }}^{\text {HSE }}$ & $\omega_{\text {AFM }}^{\Delta}$ & $\omega_{\mathrm{FM}}^{\mathrm{HSE}}$ & $\omega_{\mathrm{FM}}^{\Delta}$ & $\Delta \omega^{\mathrm{HSE}}$ & $\Delta \omega^{\Delta}$ \\
\hline $\mathrm{L}-\Gamma_{4}^{-}$ & 126 & -29 & -81 & -18 & 207 & -10 \\
$\mathrm{~S}-\Gamma_{4}^{-}$ & 272 & -11 & 186 & -3 & 86 & -8 \\
$\Gamma_{5}^{-}$ & 241 & -48 & 183 & -72 & 58 & 24 \\
$\mathrm{R}_{3}^{-}$ & 658 & 10 & 636 & 11 & 22 & -1 \\
$\mathrm{R}_{4}^{-}$ & 430 & -46 & 413 & -49 & 17 & 2 \\
$\mathrm{R}_{5}^{-}$ & -204 & -18 & -218 & -20 & 14 & 2 \\
$\mathrm{~A}-\Gamma_{4}^{-}$ & 569 & 35 & 557 & 40 & 12 & -5 \\
$\mathrm{R}_{5}^{+}$ & 437 & -11 & 448 & -14 & -11 & 3 \\
$\mathrm{R}_{2}^{-}$ & 890 & -27 & 885 & -31 & 5 & 4 \\
$\mathrm{R}_{4}^{-}$ & 160 & -20 & 157 & -21 & 3 & 1 \\
\hline \hline
\end{tabular}

constants and local Mn magnetic moments compared with HSE. As regards the metallic or insulating character, the PBEsol $+U$ agrees qualitatively with the HSE result in most cases, although the band gap is underestimated. In $\mathrm{FM}-\mathrm{SrMnO}_{3}$ there is a clear discrepancy between PBEsol $+U$ and HSE: the HSE calculations predict a half-metallic state, while we obtain a metal with PBEsol $+U$.

We have also calculated the Born effective charges for insulating AFM configurations of $\mathrm{CaMnO}_{3}\left(\mathrm{SrMnO}_{3}\right.$, $\left.\mathrm{BaMnO}_{3}\right)$. We obtain $Z_{\mathrm{Ca} / \mathrm{Sr} / \mathrm{Ba}}=2.60 e(2.58 e, 2.73 e)$, $Z_{\mathrm{Mn}}=7.35 e \quad(7.83 e, \quad 9.42 e), \quad Z_{\mathrm{O}_{\|}}=-6.55 e \quad(-6.93 e$, $-8.07 e), Z_{\mathrm{O}_{\perp}}=-1.70 e(-1.74 e,-2.04 e)$, where $e$ is the elementary charge and $Z_{\mathrm{O}_{\|}}$and $Z_{\mathrm{O}_{\perp}}$ refer, respectively, to the dynamical charges defined for an atomic displacement parallel and perpendicular to the $\mathrm{Mn}-\mathrm{O}$ bond. The anomalously large $Z_{\mathrm{Mn}}$ and $Z_{\mathrm{O}_{\|}}$charges of AFM state of $\mathrm{CaMnO}_{3}, \mathrm{SrMnO}_{3}$ and $\mathrm{BaMnO}_{3}$ are strongly reminiscent of the results obtained for ferroelectric perovskite oxides,$\frac{76}{}$ and suggest the possible proximity of a polar instability that might be triggered by an appropriate external (e.g., strain) field. $\underline{\underline{5,30,63}}$

The phonons at $\Gamma$ and $R$ for different spin orders for $\mathrm{CaMnO}_{3}, \mathrm{SrMnO}_{3}$ and $\mathrm{BaMnO}_{3}$ are shown in Tables $\Pi$, [II] and [V] respectively; the phonon modes are ordered by descending $\Delta \omega^{\mathrm{HSE}}$. The first thing to note from the table is that the frequencies obtained for $\mathrm{SrMnO}_{3}$ 's polar modes (i.e., 177, 187 and $494 \mathrm{~cm}^{-1}$ ) agree well with available low temperature experiment results (i.e., 162, 188 and $498 \mathrm{~cm}^{-1}$ taken from Ref. 77 ). Thus, our results provide additional evidence that the HSE scheme renders accurate phonon frequencies for magnetic oxides. The second thing to note from these tables is that the phononfrequency shifts $(\Delta \omega)$ obtained with PBEsol $+U$ are in overall good agreement with the HSE results, which suggests that our strategy to fit the value of $U$ is a good one. 
TABLE III: Calculated phonon frequencies and frequency shifts, as in Table II but for $\mathrm{SrMnO}_{3}$.

\begin{tabular}{lrrrrrr}
\hline \hline & $\omega_{\text {AFM }}^{\text {HSE }}$ & $\omega_{\text {AFM }}^{\Delta}$ & $\omega_{\mathrm{FM}}^{\mathrm{HSE}}$ & $\omega_{\mathrm{FM}}^{\Delta}$ & $\Delta \omega^{\mathrm{HSE}}$ & $\Delta \omega^{\Delta}$ \\
\hline $\mathrm{S}-\Gamma_{4}^{-}$ & 177 & 40 & -96 & -16 & 273 & 56 \\
$\mathrm{R}_{5}^{-}$ & 64 & -135 & -6 & -121 & 70 & -14 \\
$\Gamma_{5}^{-}$ & 292 & -38 & 253 & -54 & 39 & 16 \\
$\mathrm{R}_{2}^{-}$ & 811 & -26 & 789 & -25 & 22 & -1 \\
$\mathrm{R}_{3}^{-}$ & 571 & 12 & 553 & 2 & 18 & 10 \\
$\mathrm{R}_{4}^{-}$ & 424 & -47 & 410 & -62 & 14 & 15 \\
$\mathrm{~L}-\Gamma_{4}^{-}$ & 187 & -16 & 177 & -7 & 10 & -9 \\
$\mathrm{~A}-\Gamma_{4}^{-}$ & 494 & 22 & 487 & 17 & 7 & 5 \\
$\mathrm{R}_{5}^{+}$ & 412 & -12 & 409 & -12 & 3 & 0 \\
$\mathrm{R}_{4}^{-}$ & 159 & -6 & 157 & -7 & 2 & 0 \\
\hline \hline
\end{tabular}

Third, our results offer information about the dependence of the structural instabilities on the choice of the A-site cation and on the magnetic arrangement. We find that the AFD instability ( $R_{5}^{-}$mode) becomes weaker and disappears, for both AFM and FM states, as the size of the A-site cation increases (The effective ionic radii estimated by Shannon 78 are $r_{\mathrm{Ca}}=1.34 \AA, r_{\mathrm{Sr}}=1.44 \AA$, and $r_{\mathrm{Ba}}=1.61 \AA$.) In contrast, the ferroelectric instability (Slater mode) becomes stronger as the A-site cation becomes bigger. This is the usual behavior that one would expect for these two instabilities of the cubic perovskite structure, and has been recently examined in detail by other authors. $\frac{5,79,80}{1 t}$ clearly suggests that some of these compounds could display a magnetically ordered ferroelectric ground state. In particular, this could be the case for $\mathrm{SrMnO}_{3}$ and $\mathrm{BaMnO}_{3}$ : in these compounds, the AFD instability becomes weaker or even disappears, so that it can no longer compete with and suppress the FE soft mode.

The phonon frequency shift $\Delta \omega$ between the AFM and FM magnetic orders is shown in Fig. 5. We

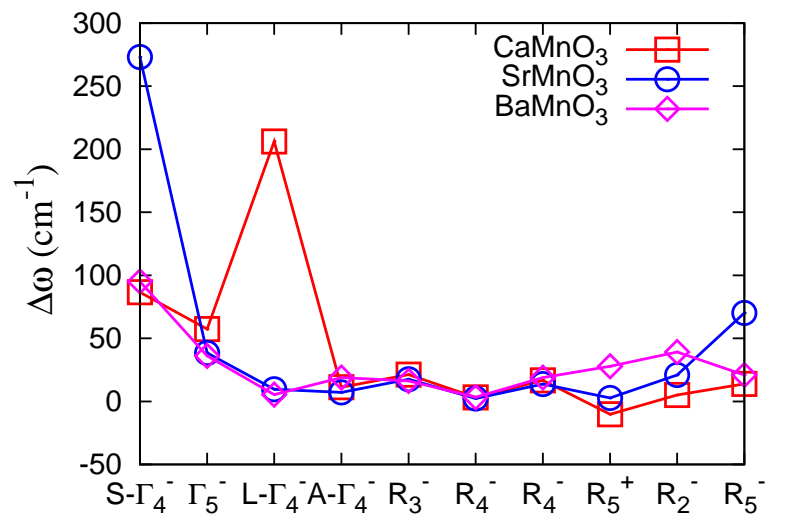

FIG. 5: (Color online) Frequency shifts $\Delta \omega=\omega_{\mathrm{AFM}}-\omega_{\mathrm{FM}}$ in $A \mathrm{MO}_{3}$ from HSE calculations.
TABLE IV: Calculated phonon frequencies and frequency shifts, as in Table II but for $\mathrm{BaMnO}_{3}$.

\begin{tabular}{lrrrrrr}
\hline \hline & $\omega_{\mathrm{AFM}}^{\mathrm{HSE}}$ & $\omega_{\mathrm{AFM}}^{\Delta}$ & $\omega_{\mathrm{FM}}^{\mathrm{HSE}}$ & $\omega_{\mathrm{FM}}^{\Delta}$ & $\Delta \omega^{\mathrm{HSE}}$ & $\Delta \omega^{\Delta}$ \\
\hline $\mathrm{S}-\Gamma_{4}^{-}$ & -274 & 117 & -369 & 227 & 95 & -110 \\
$\mathrm{R}_{2}^{-}$ & 670 & -28 & 631 & -59 & 39 & 31 \\
$\Gamma_{5}^{-}$ & 317 & -34 & 281 & -28 & 36 & -6 \\
$\mathrm{R}_{5}^{+}$ & 363 & -13 & 335 & -22 & 28 & 9 \\
$\mathrm{R}_{5}^{-}$ & 221 & -28 & 200 & -67 & 21 & 39 \\
$\mathrm{R}_{4}^{-}$ & 403 & -48 & 384 & -60 & 19 & 12 \\
$\mathrm{~A}-\Gamma_{4}^{-}$ & 423 & -12 & 404 & 11 & 19 & -22 \\
$\mathrm{R}_{3}^{-}$ & 423 & 16 & 407 & -20 & 16 & 36 \\
$\mathrm{~L}-\Gamma_{4}^{-}$ & 200 & -6 & 195 & -6 & 5 & 0 \\
$\mathrm{R}_{4}^{-}$ & 150 & -5 & 147 & -6 & 3 & 1 \\
\hline \hline
\end{tabular}

find that the Slater modes exhibit a very considerable spin-phonon coupling for all three compounds, with $\Delta \omega \gtrsim 100 \mathrm{~cm}^{-1}$. A sizable effect is also obtained for the $\Gamma_{5}^{-}$mode $\left(\Delta \omega \sim 50 \mathrm{~cm}^{-1}\right)$ in all cases. Additionally, the Last mode shows a very large effect for $\mathrm{CaMnO}_{3}$, and the spin-phonon coupling for $\mathrm{R}_{5}^{-}$is significant in the case of $\mathrm{SrMnO}_{3}$. The coupling is relatively small, or even negligible, for all other modes.

We will not attempt any detailed interpretation of our quantitative results here, but some words are in order. In the recent literature, it has often been claimed that
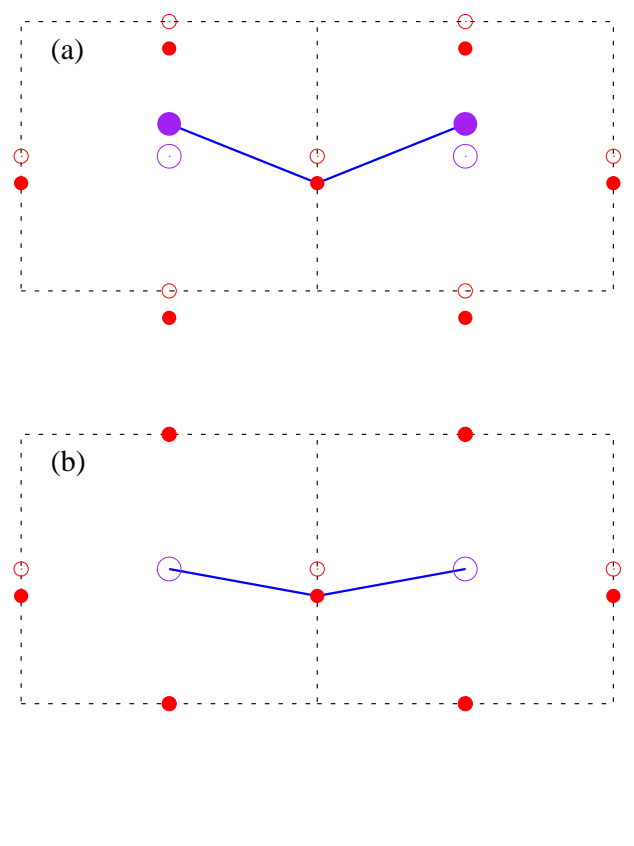

FIG. 6: (Color online) Changes to metal-O-metal bond angle (solid blue line) resulting from Slater (a) and $\Gamma_{5}^{-}$(b) phonon modes. Open and solid dots indicate ideal and displaced positions respectively. Small red dots are oxygen; larger purple dots are metal atoms. 
TABLE V: Lattice constant $a$, local magnetic moment $m$, band gap $E_{\text {gap }}$, dielectric constant $\varepsilon$, and Born effective charge $Z$ for $\mathrm{LaCrO}_{3}, \mathrm{LaFeO}_{3}$ and $\mathrm{La}_{2} \mathrm{CrFeO}_{6}(\mathrm{LCFO})$ from GGA $+U(+U)$ and $\mathrm{HSE}$ methods.

\begin{tabular}{|c|c|c|c|c|c|c|c|c|c|c|}
\hline & & $a(\AA)$ & $m\left(\mu_{\mathrm{B}}\right)$ & $E_{\text {gap }}(\mathrm{eV})$ & $\varepsilon_{\infty}$ & $\varepsilon_{0}$ & $Z_{\mathrm{La}}(e)$ & $Z_{\mathrm{Cr} / \mathrm{Fe}}(e)$ & $Z_{\mathrm{O}_{\|}}(e)$ & $\overline{Z_{\mathrm{O}_{\perp}}(e)}$ \\
\hline \multirow{2}{*}{$\mathrm{LaCrO}_{3}-\mathrm{AFM}$} & $+U$ & 3.87 & 2.86 & 2.01 & 6.12 & 93.71 & 4.53 & 3.34 & -3.50 & -2.19 \\
\hline & HSE & 3.87 & 2.75 & 3.06 & & & & & & \\
\hline \multirow{2}{*}{$\mathrm{LaCrO}_{3}-\mathrm{FM}$} & $+U$ & 3.88 & 2.84 & 1.70 & 6.08 & 236.19 & 4.52 & 3.61 & -3.62 & -2.26 \\
\hline & HSE & 3.88 & 2.74 & 2.49 & & & & & & \\
\hline \multirow{2}{*}{$\mathrm{LaFeO}_{3}-\mathrm{AFM}$} & $+U$ & 3.90 & 4.19 & 2.20 & 6.37 & & 4.47 & 3.75 & -3.41 & -2.43 \\
\hline & HSE & 3.91 & 4.10 & 3.25 & & & & & & \\
\hline \multirow{2}{*}{$\mathrm{LaFeO}_{3}-\mathrm{FM}$} & $+U$ & 3.91 & 4.38 & 1.57 & 6.19 & & 4.48 & 4.02 & -3.59 & -2.46 \\
\hline & HSE & 3.93 & 4.26 & 2.20 & & & & & & \\
\hline \multirow{2}{*}{ LCFO-AFM } & $+U$ & 3.88 & $2.71 / 4.30$ & 1.98 & 6.35 & 713.57 & 4.51 & $3.02 / 4.22$ & -3.50 & -2.33 \\
\hline & HSE & 3.88 & $2.63 / 4.19$ & 2.78 & & & & & & \\
\hline \multirow{2}{*}{ LCFO-FM } & $+U$ & 3.89 & $3.01 / 4.28$ & 2.01 & 6.18 & 262.40 & 4.49 & $3.21 / 3.87$ & -3.45 & -2.30 \\
\hline & HSE & 3.89 & $2.89 / 4.18$ & 3.04 & & & & & & \\
\hline
\end{tabular}

AFD distortions are expected to couple strongly with the magnetic structure of perovskite oxides, as they control the metal-oxygen-metal angles that are critical to determine the magnitude of the magnetic interactions that are dominant in insulators ${ }^{15}$ [The hopping parameters between oxygen $(2 p)$ and transition metal $(3 d)$ orbitals are strongly dependent on such angles, as are the effective hoppings between $3 d$ orbitals of neighboring transition metals. As we know form the GoodenoughKanamori-Anderson rules, the super-exchange interactions are strongly dependent on such hoppings.] In principle, such an effect should be reflected in our computed frequency shifts; for such $R_{5}^{-}$modes we obtain $\Delta \omega$ values ranging between $10 \mathrm{~cm}^{-1}$ and $70 \mathrm{~cm}^{-1}$. Interestingly, following the same argument, one would conclude that the Slater and $\Gamma_{5}^{-}$modes should have a similar impact on the magnetic couplings in these materials, as both involve changes in the metal-oxygen-metal angles due to the relative displacement of metal and oxygen atoms, as shown in Fig. 6. Further, the Slater modes also affect the metaloxygen distances, as they involve a significant shortening of some metal-oxygen bonds (Fig. [6 ). Hence, from this perspective, the large $\Delta \omega$ values obtained in our calculations for these two types of modes are hardly surprising. Finally, the result obtained for the Last mode in the case of $\mathrm{CaMnO}_{3}$ (i.e., $\Delta \omega \approx 200 \mathrm{~cm}^{-1}$ ) is clearly anomalous and unexpected according to the above arguments. We speculate that it may be related to a significant $\mathrm{Ca}-\mathrm{O}$ interaction that alters the usual super-exchange mechanism and favors a FM interaction. While unusual, effects that seem similar have been reported previously for other compounds,$\underline{81,82}$

\section{Spin-phonon coupling in $\mathrm{LaCrO}_{3}, \mathrm{LaFeO}_{3}$, and $\mathrm{La}_{2} \mathrm{CrFeO}_{6}$}

In the previous Section we have shown how spinphonon coupling effects can crucially depend on the nature of the $A$-site cations in $A_{M n O}$ compounds. Now we will focus on the change of $B$-site cation to investigate the spin-phonon couplings in the $\mathrm{La} \mathrm{O}_{3}$ compounds $(M=\mathrm{Cr}, \mathrm{Fe})$ and the double perovskite $\mathrm{La}_{2} \mathrm{CrFeO}_{6}$. For our PBEsol $+U$ calculations we used $U=3.8 \mathrm{eV}$ for $\mathrm{Cr}$ and $U=5.1 \mathrm{eV}$ for $\mathrm{Fe}$, which were determined as described in Section [II. We obtained these $U$ 's from calculations for $\mathrm{LaCrO}_{3}$ and $\mathrm{LaFeO}_{3}$ and used the same values for the PBEsol $+U$ study of $\mathrm{La}_{2} \mathrm{CrFeO}_{6}$.

The basic computed properties of $\mathrm{LaCrO}_{3}, \mathrm{LaFeO}_{3}$ and $\mathrm{La}_{2} \mathrm{CrFeO}_{6}$ are presented in Table $\mathrm{V}$ In all cases, we obtain insulating solutions for AFM and FM spin orders, both from HSE and PBEsol $+U$ calculations. Therefore, we also calculated the Born effective charges and optical dielectric constants within PBEsol $+U$ by using densityfunctional perturbation theory as implemented in VASP. The static dielectric constants were also calculated for compounds which do not have an unstable polar mode. It is evident that $Z_{\mathrm{La}}$ and $Z_{\mathrm{O}_{\perp}}$ are essentially identical for the three compounds and insensitive to the spin order. On the other hand, the $Z_{\mathrm{Cr} / \mathrm{Fe}}$ and $Z_{\mathrm{O}_{\|}}$charges of $\mathrm{LaCrO}_{3}$ and $\mathrm{LaFeO}_{3}$ increase in magnitude when the spin arrangement changes from AFM to FM, and de-

TABLE VI: Calculated phonon frequencies and frequency shifts, as in Table II but for $\mathrm{LaCrO}_{3}$.

\begin{tabular}{lrrrrrr}
\hline \hline & $\omega_{\mathrm{AFM}}^{\mathrm{HSE}}$ & $\omega_{\mathrm{AFM}}^{\Delta}$ & $\omega_{\mathrm{FM}}^{\mathrm{HSE}}$ & $\omega_{\mathrm{FM}}^{\Delta}$ & $\Delta \omega^{\mathrm{HSE}}$ & $\Delta \omega^{\Delta}$ \\
\hline$\Gamma_{5}^{-}$ & 230 & -24 & 178 & -35 & 52 & 11 \\
$\mathrm{~S}-\Gamma_{4}^{-}$ & 361 & -26 & 317 & -27 & 44 & 1 \\
$\mathrm{~L}-\Gamma_{4}^{-}$ & 69 & 0 & 48 & -3 & 21 & 3 \\
$\mathrm{R}_{4}^{-}$ & 389 & -29 & 372 & -30 & 17 & 1 \\
$\mathrm{R}_{5}^{+}$ & 414 & 2 & 430 & 0 & -16 & 2 \\
$\mathrm{~A}-\Gamma_{4}^{-}$ & 659 & 32 & 644 & 30 & 15 & 2 \\
$\mathrm{R}_{5}^{-}$ & -213 & 1 & -228 & 1 & 15 & 0 \\
$\mathrm{R}_{3}^{-}$ & 683 & 5 & 669 & 5 & 14 & 0 \\
$\mathrm{R}_{4}^{-}$ & 89 & -4 & 81 & -4 & 8 & 0 \\
$\mathrm{R}_{2}^{-}$ & 840 & -17 & 843 & -19 & -3 & 2 \\
\hline \hline
\end{tabular}


crease in the case of the double perovskite $\mathrm{La}_{2} \mathrm{CrFeO}_{6}$. Table $\nabla$ also shows that the optical dielectric constants $\varepsilon_{\infty}$ are very close to 6 for these three materials, and are independent of the magnetic order. However, the static dielectric constants $\varepsilon_{0}$ of $\mathrm{LaCrO}_{3}$ and $\mathrm{La}_{2} \mathrm{CrFeO}_{6}$ change very significantly when moving from AFM to FM. Also, the static dielectric constant of $\mathrm{AFM}-\mathrm{La}_{2} \mathrm{CrFeO}_{6}$ is very large due to the very small frequency of the Last phonon mode. The static dielectric constants of $\mathrm{LaFeO}_{3}$ are not shown in Table $\mathrm{V}$ because the Last modes are unstable, and strictly speaking they are not well defined. (Roughly speaking, in all such cases we would have $\varepsilon_{0} \rightarrow \infty$, as the cubic phase is unstable with respect to a polar distortion.)

In Tables VI and VII] we show the phonon frequencies as calculated at the HSE and PBEsol $+U$ levels for $\mathrm{LaCrO}_{3}$ and $\mathrm{LaFeO}_{3}$ respectively. Further, Table VIII shows the PBEsol $+U$ results for $\mathrm{La}_{2} \mathrm{CrFeO}_{6}$. As was the case for the $\mathrm{AMnO}_{3}$ compounds of the previous section, we find here as well that the AFM-FM frequency shifts computed with our PBEsol $+U$ scheme reproduce well the HSE results.

Our results also show that the phonons of the $\mathrm{LaMO}_{3}$ compounds exhibit some features that differ from those of the $\mathrm{AMnO}_{3}$ materials. First, the octahedral rotation mode $\left(\mathrm{R}_{5}^{-}\right)$is unstable for all the La-based compounds, and it is largely insensitive to the nature of the $B$-site cation. Second, the energetics of the FE modes is very different. In the case of the Mn-based compounds, the Slater mode is the lowest-frequency mode, and in some cases it becomes unstable, thus inducing a polarization, just as the unstable Slater mode induces ferroelectricity in $\mathrm{BaTiO}_{3} \underset{70}{\underline{70}}$ However, in the La compounds the lowestfrequency mode is the Last phonon mode, and in the cases of $\mathrm{LaFeO}_{3}$ and $\mathrm{La}_{2} \mathrm{CrFeO}_{6}$ this Last mode is unstable and might induce ferroelectricity; this situation is more analogous to what occurs in $\mathrm{PbTiO}_{3} \underline{70}$ or $\mathrm{BiFeO}_{3}$.

The spin-phonon coupling effects computed for the $\mathrm{LaMO}_{3}$ compounds are given in Tables VI, VII and VIII. and are summarized in Fig. 7 Here, the first thing to note is that the magnitude of the effects is significantly

TABLE VII: Calculated phonon frequencies and frequency shifts, as in Table II but for $\mathrm{LaFeO}_{3}$.

\begin{tabular}{lrrrrrr}
\hline \hline & $\omega_{\text {AFM }}^{\text {HSE }}$ & $\omega_{\text {AFM }}^{\Delta}$ & $\omega_{\text {FM }}^{\text {HSE }}$ & $\omega_{\mathrm{FM}}^{\Delta}$ & $\Delta \omega^{\text {HSE }}$ & $\Delta \omega^{\Delta}$ \\
\hline $\mathrm{R}_{2}^{-}$ & 796 & -38 & 822 & -40 & -26 & 2 \\
$\mathrm{~A}_{-} \Gamma_{4}^{-}$ & 645 & -7 & 630 & -4 & 15 & -3 \\
$\mathrm{~S}_{-} \Gamma_{4}^{-}$ & 259 & 4 & 247 & 4 & 12 & 0 \\
$\mathrm{R}_{3}^{-}$ & 549 & -11 & 536 & -8 & 13 & -3 \\
$\mathrm{R}_{4}^{-}$ & 390 & -24 & 379 & -23 & 11 & -1 \\
$\mathrm{~L}_{-} \Gamma_{4}^{-}$ & -81 & 12 & -92 & 9 & 11 & 3 \\
$\mathrm{R}_{5}^{-}$ & -237 & 9 & -247 & 7 & 10 & 2 \\
$\mathrm{R}_{4}^{-}$ & 66 & -2 & 56 & -2 & 10 & 0 \\
$\mathrm{R}_{5}^{+}$ & 346 & -5 & 355 & -6 & -9 & 1 \\
$\Gamma_{5}^{-}$ & 120 & -3 & 115 & -10 & 5 & 7 \\
\hline \hline
\end{tabular}

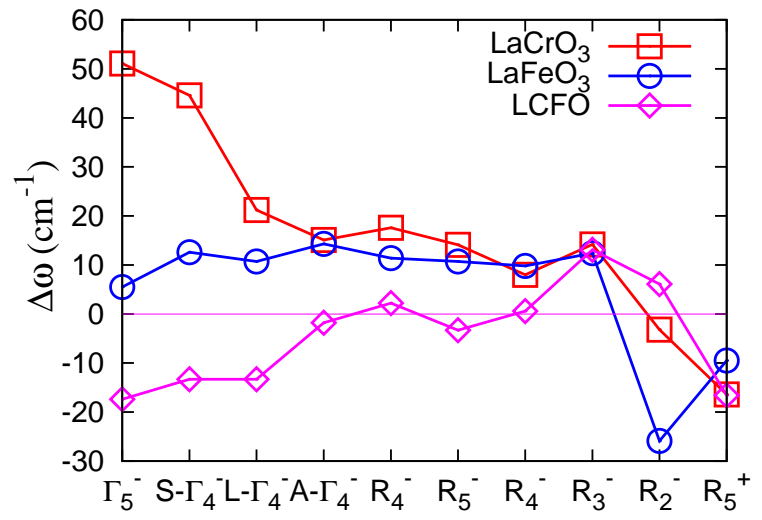

FIG. 7: (Color online) Frequency shifts for $\mathrm{LaMO}_{3}$ (HSE for $\mathrm{LaCrO}_{3}$ and $\mathrm{LaFeO}_{3} ; \mathrm{PBEsol}+U$ for $\left.\mathrm{La}_{2} \mathrm{CrFeO}_{6}\right)$.

smaller than for the $\mathrm{La} \mathrm{O}_{3}$ compounds (note the different scales of Figs. 5 and (7). Second, we observe that the largest effects are associated with the $\Gamma_{5}^{-}$and Slater modes (with $\Delta \omega \approx 45 \mathrm{~cm}^{-1}$ for $\mathrm{LaCrO}_{3}$ ). This is consistent with the point made above that these modes disrupt the metal-oxygen-metal super-exchange paths. In comparison, in this case we obtain a relatively small effect for the $\mathrm{R}_{5}^{-}$modes, which show $\Delta \omega$ values $\left(\lesssim 20 \mathrm{~cm}^{-1}\right)$ that are comparable to those computed for most of the phonon modes considered. Finally, for $\mathrm{LaCrO}_{3}$ and $\mathrm{LaFeO}_{3}$ we observe that the $\Gamma$ phonon frequencies decrease as the spin order changes from AFM to FM, in line with what was observed for the $A \mathrm{MnO}_{3}$ compounds. In contrast, the $\Gamma$ modes of double-perovskite $\mathrm{La}_{2} \mathrm{CrFeO}_{6}$ increase in frequency when the spin order changes to FM.

\section{Prospects for the design of new multiferroics}

Let us now discuss several possible implications of our results regarding the design of novel multiferroic materi-

TABLE VIII: Calculated phonon frequencies and frequency shifts, as in Table II but for $\mathrm{La}_{2} \mathrm{CrFeO}_{6}$ from $\mathrm{PBEsol}+U^{83}$

\begin{tabular}{lrrr}
\hline \hline & $\omega_{\mathrm{AFM}}$ & $\omega_{\mathrm{FM}}$ & $\Delta \omega$ \\
\hline $\mathrm{L}-\Gamma_{4}^{-}$ & 31 & 45 & -14 \\
$\Gamma_{5}^{-}$ & 161 & 178 & -17 \\
$\mathrm{R}_{5}^{+}$ & 383 & 400 & -17 \\
$\mathrm{~S}_{-} \Gamma_{4}^{-}$ & 284 & 297 & -13 \\
$\mathrm{R}_{3}^{-}$ & 629 & 616 & 13 \\
$\mathrm{R}_{2}^{-}$ & 803 & 797 & 6 \\
$\mathrm{R}_{5}^{-}$ & -225 & -222 & -3 \\
$\mathrm{R}_{4}^{-}$ & 362 & 360 & 2 \\
$\mathrm{~A}-\Gamma_{4}^{-}$ & 674 & 676 & -2 \\
$\mathrm{R}_{4}^{-}$ & 79 & 79 & 0 \\
\hline \hline
\end{tabular}


als.

\section{1. $\mathrm{BaMnO}_{3}$-based materials}

As already mentioned in the Introduction, the spinphonon coupling can trigger multiferroicity in some materials if, by application of an epitaxial strain or other perturbation, a FM-FE state can be stabilized with respect to the AFM-PE ground state ${ }^{22}$ Indeed, the three investigated $\mathrm{AMnO}_{3}$ compounds have been suggested as candidates to exhibit strain-induced multiferroicity by

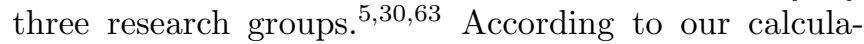
tions, we can tentatively suggest that $\mathrm{BaMnO}_{3}$ might show multiferroicity even without strain applied, provided the material can be grown as a distorted perovskite (the most stable polymorph of $\mathrm{BaMnO}_{3}$ adopts instead a structure with face-sharing octahedra 63,84 . Note that it may be possible to enhance the stability of $\mathrm{BaMnO}_{3}$ 's cubic perovskite phase by partial substitution of $\mathrm{Ba}$ by $\mathrm{Ca}$ or Sr. In fact, ideally one would try to obtain samples of $\mathrm{Ba}_{1-x} \mathrm{Sr}_{x} \mathrm{MnO}_{3}$ or $\mathrm{Ba}_{1-x} \mathrm{Ca}_{x} \mathrm{MnO}_{3}$ with $x$ large enough to stabilize the perovskite phase, and small enough for the FE instability associated to the FM Slater mode to dominate over the $\mathrm{R}_{5}^{-}$and AFM Slater modes. Apparently this strategy to obtain new multiferroics has recently been realized experimentally by Sakai et al $\frac{85}{i n}$ $\mathrm{Ba}_{1-x} \mathrm{Sr}_{x} \mathrm{MnO}_{3}$ solid solutions. An alternative would be to consider $\mathrm{CaMnO}_{3} / \mathrm{BaMnO}_{3}$ or $\mathrm{SrMnO}_{3} / \mathrm{BaMnO}_{3}$ superlattices with varying ratios of the pure compounds, and perhaps tuning the misfit strain via the choice of substrate.

Another intriguing possibility pertains to the magnetic response of the $\mathrm{AMnO}_{3}$ compounds that display an AFM-PE ground state and a dominant polar instability of their FM phase. Again, this could be the case for some $\mathrm{Ba}_{1-x} \mathrm{Sr}_{x} \mathrm{MnO}_{3}$ and $\mathrm{Ba}_{1-x} \mathrm{Ca}_{x} \mathrm{MnO}_{3}$ solid solutions with an appropriate choice of $x$. By applying a magnetic field to such compounds, it might be possible to switch them from the AFM ground state to a FM spin configuration and, as a result, induce a ferroelectric polarization.

\section{Double-perovskite $\mathrm{La}_{2} \mathrm{CrFeO}_{6}$}

The double perovskite $\mathrm{La}_{2} \mathrm{CrFeO}_{6}$ has been intensively studied to examine its possible magnetic order through $3 d^{3}-3 d^{5}$ superexchange. However, its magnetic ground state has long been debated. Pickett et al ${ }^{86}$ predicted that the ferrimagnetic (FiM) ground state with a net spin moment of $2 \mu_{\mathrm{B}} /$ f.u. is more stable than the FM one with $7 \mu_{\mathrm{B}} /$ f.u. (This FiM order can be viewed as a G-AFM configuration in which, for example, all $\mathrm{Fe}$ spins are pointing up and all $\mathrm{Cr}$ spins are pointing down.) However, from GGA and $\mathrm{LDA}+U$ calculations, Miura et al. found that the ground-state magnetic ordering of $\mathrm{La}_{2} \mathrm{CrFeO}_{6}$ is FiM in GGA, but that even a small $U$ in $\mathrm{LDA}+U$ makes it $\mathrm{FM} .87$ The experimental picture is also unclear. Ueda et al. have grown a (111)-oriented $\mathrm{LaCrO}_{3} / \mathrm{LaFeO}_{3}$ superlattice which exhibits FM ordering, although the measured saturation magnetization is much smaller than expected $\$ 8$ Very recently, Chakraverty et al. reported epitaxial $\mathrm{La}_{2} \mathrm{CrFeO}_{6}$ double-perovskite films grown by pulsed-laser deposition, and their sample exhibits FiM with a saturation magnetization of $2.0 \pm 0.15 \mu_{\mathrm{B}} /$ f.u. at $5 \mathrm{~K} \underline{89}$

Our HSE calculations for $\mathrm{La}_{2} \mathrm{CrFeO}_{6}$ with the atomic (oxygen) positions relaxed in the cubic structure shows the magnetic ground state has a FiM spin pattern leading to a FiM structure with a net magnetization of $1.56 \mu_{\mathrm{B}} /$ f.u. This is consistent with $\mathrm{LDA}^{86}$ and GGA ${ }^{87}$ calculations, as well as being heuristically consistent with the experimental report of $\mathrm{AFM}$ ordering in $\mathrm{La}_{2} \mathrm{CrFeO}_{6}$ solid-solution films $\underline{89}$ However, our HSE calculation shows that the energy difference between the FiM and FM states is very small: FiM is only $0.8 \mathrm{meV} /$ f.u. lower in energy than FM. In addition, the GGA $+U$ with $U$ fitted to $\mathrm{LaCrO}_{3}$ and $\mathrm{LaFeO}_{3}(U=3.8$ and $5.1 \mathrm{eV}$ for $\mathrm{Cr}$ and $\mathrm{Fe}$, respectively) results in a FM magnetic ground state having a total energy $34.9 \mathrm{meV}$ lower than that of the FiM. By doing a fitting of the $U$ parameters for $\mathrm{Cr}$ and Fe directly to $E_{\mathrm{AFM}}-E_{\mathrm{FM}}$ of $\mathrm{La}_{2} \mathrm{CrFeO}_{6}$ as computed by PBEsol $+U$ and HSE, instead of for $\mathrm{LaCrO}_{3}$ and $\mathrm{LaFeO}_{3}$ separately, we find parameters of $U=3.0$ and $4.1 \mathrm{eV}$ for $\mathrm{Cr}$ and Fe respectively (the phonon properties predicted from these are very close to our previous results). Using these parameters, we find that the FiM ground state is $0.7 \mathrm{meV}$ lower in energy than the FM state. For comparison, a straight PBEsol calculation (with $U=0$ ) predicts that the energy of the FiM ground state is $596 \mathrm{meV} /$ f.u. lower than that of the FM state. Clearly, $U$ should be chosen carefully in order to obtain the correct ground state of $\mathrm{La}_{2} \mathrm{CrFeO}_{6}$.

According to our HSE calculations, the magnetic ground state of $\mathrm{La}_{2} \mathrm{CrFeO}_{6}$ is FiM, but with the FM state lying only very slightly higher in energy. This may be the reason why questions about the magnetic ground state of $\mathrm{La}_{2} \mathrm{CrFeO}_{6}$ have long been debated; the energy difference is so small that external perturbations (e.g., the epitaxial strain in a superlattice ${ }^{88}$ ) or variations in $U$ between different $\mathrm{LDA}+U^{87}$ and $\mathrm{GGA}+U$ calculations may bring the FM energy below that of the AFM. Taken together with our results, shown in Table VIII, that the Last mode is close to going soft in this material, this suggests that $\mathrm{La}_{2} \mathrm{CrFeO}_{6}$ might be a good candidate for a material in which multiferroic phase transitions could be induced, similar to what was shown for $\mathrm{SrMnO}_{3}{ }^{30}$ and $\mathrm{SrCoO}_{3} .27$ Because the spin ordering is so delicate, it seems likely that a small misfit strain could be enough to trigger such a transition. 


\section{SUMMARY AND CONCLUSIONS}

In this work, we have studied the spin-phonon coupling for transition-metal oxides within density-functional theory. From the computational point of view, an accurate description of the electronic, structural, and vibrational properties on an equal footing is a prerequisite for a reliable study of the coupling between spins and phonon. Taking note of the increasing evidence that hybrid functionals are suitable for this task, we have adopted the Heyd-Scuseria-Ernzerhof (HSE) screened hybrid functional for the present work. However, the accuracy of the HSE results comes at the cost of an increase of computational load, so that a full frozen-phonon calculation of the phonon modes remains prohibitively expensive in many cases. We propose to overcome this limitation by carrying out calculations at the $\mathrm{DFT}+U$ level using $U$ parameters that have been fitted to HSE results for totalenergy differences between spin configurations. Our results show that the resulting $\mathrm{DFT}+U$ scheme reproduces the HSE results very accurately, especially in regard to the spin-phonon couplings of interest here.

As regards the direct HSE phonon calculations, we have developed an approach in which we split the calculation into separate, simultaneous frozen-phonon calculations for different symmetry-adapted displacement patterns, and then combine the results to calculate and diagonalize the dynamical matrix, thus accelerating these calculations significantly.

Our important results can be summarized as follows. First, we have shown that the choice of $U$ is a big concern in such studies, since the spin-phonon coupling can depend very strongly on $U$. As an alternative to extracting $U$ from experimental studies, we propose here to obtain it by fitting to HSE calculations as illustrated above. Second, we have studied $\mathrm{CaMnO}_{3}, \mathrm{SrMnO}_{3}$ and $\mathrm{BaMnO}_{3}$, focusing on trends in the spin-phonon coupling due to the increase of the $A$ cation size. Based on the strain couplings and the spin-phonon interactions, we suggest theoretically that $\mathrm{BaMnO}_{3}$ is more likely to show ferroelectricity under tensile strain and, furthermore, that A-site substitution by a cation with smaller size may induce multiferroicity even without external strain. Third, we find that in the $A \mathrm{MnO}_{3}$ materials class with $A=\mathrm{Ca}$, $\mathrm{Sr}$, and $\mathrm{Ba}$, the frequency shift decreases as the $\mathrm{A}$ cation radius increases for the $\Gamma$ phonons, while it increases for R-point phonons. Fourth, we have shown that changing B-site cations may also have important effects on the dielectric properties: in $\mathrm{La} \mathrm{O}_{3}$ with $M=\mathrm{Cr}, \mathrm{Fe}$, and $\mathrm{Cr} / \mathrm{Fe}$, the phonon frequencies at $\Gamma$ decrease as the spin order changes from AFM to FM for $\mathrm{LaCrO}_{3}$ and $\mathrm{LaFeO}_{3}$, but they increase for the double perovskite $\mathrm{La}_{2}(\mathrm{CrFe}) \mathrm{O}_{6}$. Finally, we have shown that the polar phonon modes of the investigated perovskites tend to display the largest spin-phonon couplings, while modes involving rotations of the $\mathrm{O}_{6}$ octahedra present considerable, but generally smaller, effects. Such observations may be relevant as regards current efforts to obtain large magnetostructural (and magnetoelectric) effects.

We hope that our study will stimulate further work leading to rational design and strain engineering of multiferroicity using spin-phonon couplings.

\section{Appendix: Efficient phonon calculations with hybrid functionals}

Even though we only have a 10-atom cell, we found that it can be quite expensive to use the HSE functional to carry out the needed spin-polarized calculations of phonon properties ${ }^{75}$ We overcome this limitation as follows. First, we use symmetry to limit ourselves to sets of displacements that will block-diagonalize the forceconstant matrix. For example, for the polar modes we move the cations along $x$ and the $\mathrm{O}$ atoms along $x$ and $y$. We then carry out self-consistent calculations on these displaced geometries, and from the forces we construct the relevant block of the force-constant matrix. Second, while the standard VASP implementation uses a "central difference" method in which ions are displaced by small amounts in both positive and negative displacements, we save some further effort by displacing only in the positive direction. Finally, we note that the forces resulting from each pattern of atomic displacements can be calculated independently, allowing us to split the calculation in parallel across independent groups of processors and thus further reduce the wall-clock time.

We have checked the accuracy of this approach for FM$\mathrm{LaCrO}_{3}$ using PBEsol $+U(U=3.8 \mathrm{eV})$ and for G-AFM$\mathrm{BaMnO}_{3}$ using HSE. For each case, we compared the results of the standard implementation of the VASP frozenion calculation of phonon frequencies with the revised approach described above. We take the ion displacements to be $0.015 \AA$ in all cases. We find that the RMS error of ten different phonon frequencies is $1.2 \mathrm{~cm}^{-1}$ for $\mathrm{PBEsol}+U$ and $7.3 \mathrm{~cm}^{-1}$ for HSE. These results suggest that this method has acceptable accuracy with reduced computational cost. We propose that it could be used also for cases of lower symmetry and larger cells, thus making the HSE phonon calculations at $\Gamma$ affordable in general.

Recently, a revised Perdew-Burke-Ernzerhof functional base on PBEsol, which we refer to as HSEsol, was designed to yield more accurate equilibrium properties for solids and their surfaces. Compared to HSE, significant improvements were found for lattice constants and atomization energies of solids .90 We also checked the effect of using HSEsol on our calculations, as shown in Table IX From this table, we can see that the phonons calculated with HSEsol are very close to those from HSE.

\section{Acknowledgments}

This work was supported by ONR Grant 00014-050054, by Grant Agreement No. 203523-BISMUTH of the 
TABLE IX: Comparison of phonon frequencies $\left(\mathrm{cm}^{-1}\right)$ of $\mathrm{SrMnO}_{3}$ calculated from HSE and HSEsol (sol).

\begin{tabular}{lrrrrrr}
\hline \hline & $\omega_{\text {AFM }}^{\text {HSE }}$ & $\omega_{\text {AFM }}^{\text {sol }}$ & $\omega_{\text {FM }}^{\text {HSE }}$ & $\omega_{\text {FM }}^{\text {sol }}$ & $\Delta \omega^{\text {HSE }}$ & $\Delta \omega^{\text {sol }}$ \\
\hline $\mathrm{S}-\Gamma_{4}^{-}$ & 177 & 166 & -96 & -129 & 273 & 295 \\
$\Gamma_{5}^{-}$ & 292 & 290 & 253 & 250 & 38 & 40 \\
$\mathrm{~L}_{-} \Gamma_{4}^{-}$ & 187 & 179 & 177 & 172 & 10 & 7 \\
$\mathrm{~A}-\Gamma_{4}^{-}$ & 494 & 485 & 487 & 477 & 7 & 8 \\
$\mathrm{R}_{4}^{-}$ & 159 & 156 & 157 & 154 & 2 & 3 \\
$\mathrm{R}_{5}^{+}$ & 412 & 408 & 409 & 405 & 3 & 3 \\
$\mathrm{R}_{4}^{-}$ & 424 & 419 & 410 & 406 & 14 & 14 \\
$\mathrm{R}_{3}^{-}$ & 571 & 558 & 553 & 539 & 18 & 19 \\
$\mathrm{R}_{5}^{-}$ & 64 & 71 & -6 & 26 & 70 & 45 \\
$\mathrm{R}_{2}^{-}$ & 811 & 803 & 789 & 782 & 21 & 20 \\
\hline \hline
\end{tabular}

EU-FP7 European Research Council, and by MICINNSpain (Grants No. MAT2010-18113, No. MAT201010093-E, and No. CSD2007-00041). Computations were carried out at the Center for Piezoelectrics by Design. J.H. acknowledges travel support from AQUIFER Programs funded by the International Center for Materials Research at UC Santa Barbara. We thank Jun Hee Lee, Claude Ederer and Karin Rabe for useful discussions.
* Electronic address: hongjw10@physics.rutgers.edu

1 R. Ramesh, N. A. Spaldin, Nat. Mater. 621 (2007).

2 Y.-H. Chu, L.W. Martin, M. B. Holcomb,M. Gajek, S.-J. Han,Q. He, N. Balke, C.-H. Yang,D. Lee,W. Hu,Q. Zhan, P.-L. Yang, A. Fraile-Rodríguez, A. Scholl, S. X. Wang, R. Ramesh, Nat. Mater. 7, 478 (2008).

3 M. Gajek, M. Bibes, S. Fusil, K. Bouzehouane, J. Fontcuberta, A. Barthélémy, A. Fert, Nat. Mater. 6296 (2007).

4 N. A. Hill, J. Phys. Chem. B 104, 6694 (2000).

5 S. Bhattacharjee, E. Bousquet, and P. Ghosez, Phys. Rev. Lett. 102, 117602 (2009).

6 D. Khomskii, Physics 2, 20 (2009).

7 C. Ederer, T. Harris and Roman Kováčik Phys. Rev. B 83, 054110 (2011)

8 G. Rogez, N. Viart, and M. Drillon, Angew. Chem. Int. Ed. 49, 1921 (2010).

${ }^{9}$ R. Ramesh, Nature 461, 1218 (2009).

10 A. Stroppa, P. Jain, P. Barone, M. Marsman, J. M. PerezMato, A. K. Cheetham, H. W. Kroto, S. Picozzi Angew. Chemie Int. Ed. 50, 5847 (2011).

11 A. P. Levanyuk and D. G. Sannikov, Usp. Fiz. Nauk. 112, 561 (1974).

12 J. M. Perez-Mato, M. Aroyo, A. Garcia, P. Blaha, K. Schwarz, J. Schweifer and K. Parlinski, Phys. Rev. B 79, 214111 (2004).

13 E. Bousquet, M. Dawber, N. Stucki, C. Lichtensteiger, P. Hermet, S. Gariglio, J.-M. Triscone and P. Ghosez, Nature, 452, 732 (2008).

14 C. J. Fennie and K. M. Rabe, Phys. Rev. B 72, 100103(R) 2005.

15 N. A. Benedek and C. J. Fennie, Phys. Rev. Lett. 106, 107204 (2011).

16 P. Ghosez and J. M. Triscone, Nat. Mater., 10, 269 (2011).

17 G. Lawes, Physics, 4, 18 (2011)

18 C. Ederer and N. Spaldin, Phys. Rev. B 74, 024102 (2006).

19 Jorge Lopez-Prez and Jorge Iniguez, Phys. Rev. B 84, 075121 (2011).

${ }^{20}$ R. E. Cohen, Nature (London) 358, 136 (1992).

21 J. H. Haeni, P. Irvin, W. Chang, R. Uecker, P. Reiche, Y. L. Li, S. Choudhury, W. Tian, M. E. Hawley, B. Craigo, A. K. Tagantsev, X. Q. Pan, S. K. Streiffer, L. Q. Chen, S. W. Kirchoefer, J. Levy and D. G. Schlom, Nature, 430,
758 (2004).

22 C. J. Fennie and K. M. Rabe, Phys. Rev. Lett. 97267602 (2006).

23 J. H. Lee, L. Fang, E. Vlahos, X. Ke, Y. W. Jung, L. F. Kourkoutis, J.-W. Kim, P. J. Ryan, T. Heeg, M. Roeckerath Nature 466, 954 (2010).

24 J. C. Wojdel and J. Iniguez Phys. Rev. Lett. 105, 037208 (2010).

25 J. M. Rondinelli and N. A. Spaldin, Adv. Mat. 23, 3363 (2011).

26 J. H. Lee and K. M. Rabe Phys. Rev. B 84, 104440 (2011).

27 J. H. Lee and K. M. Rabe Phys. Rev. Lett. 107, 067601 (2011).

28 M. Mochizuki, N. Furukawa, and N. Nagaosa, Phys. Rev. B 84, 144409 (2011).

${ }^{29}$ H. Das, U. V. Waghmare, T. Saha-Dasgupta, and D. D. Sarma, Phys. Rev. Lett. 100, 186402 (2008).

30 Jun Hee Lee and Karin M. Rabe, Phys. Rev. Lett. 104, 207204 (2010).

31 J. Hemberg, T. Rudolf, H.-A. Krug von Nidda, F. Mayr, A. Pimenov, V. Tsurkan and A. Loidl1, Phys. Rev. Lett. 97, 087204 (2006).

32 T. Rudolf, C. Kant, F. Mayr and A. Loidl, Phys. Rev. B 77, $024421(2008)$

33 S. Kümmel and L. Kronik, Rev. Mod. Phys. 80, 3 (2008).

34 C. Loschen, J. Carrasco, K. M. Neyman, and F. Illas, Phys. Rev. B 75, 035115 (2007).

35 A. Rohrbach, J. Hafner and G. Kresse, J. Phys. Cond. Mat. 15, 979 (2003).

36 A. Stroppa and S. Picozzi, Phys. Chem. Chem. Phys. 12, 5405 (2010).

37 Nirat Ray and Umesh V. Waghmare, Phys. Rev. B 77, 134112 (2008).

38 J. Heyd, J. E. Peralta, G. E. Scuseria, and R. L. Martin, J. Chem. Phys. 123, 174101 (2005).

39 J. Paier, R. Hirschl, M. Marsman, and G. Kresse, J. Chem. Phys. 122, 234102 (2005); J. Paier, M. Marsman, K. Hummer, G. Kresse, I. C. Gerber, and J. G. Ángyán, ibid. 124, 154709 (2006); J. Paier, M. Marsman, and G. Kresse, ibid. 127, 024103 (2007); M. Marsman, J. Paier, A. Stroppa, and G. Kresse, J. Phys.: Condens. Matter 20, 064201 (2008); A. Stroppa and G. Kresse, New J. Phys. 10, 063020 (2008). 
40 J. Paier, M. Marsman, and G. Kresse, Phys. Rev. B 78, 121201 (R) (2008).

41 D. O. Scanlon and G. Watson, Chem. Mater. 21, 5435 (2009).

42 D. O. Scanlon, B. J. Morgan, G. W. Watson, and A. Walsh, Phys. Rev. Lett. 103, 096405 (2009).

43 D. O. Scanlon, A. Walsh, and G. Watson, Chem. Mater 21, 4568 (2009).

44 J. P. Allen, D. O. Scanlon, and G. W. Watson, Phys. Rev. B 81, 161103(R) (2010).

45 F. Oba, A. Togo, I. Tanaka, J. Paier, and G. Kresse, Phys. Rev. B 77, 245202 (2008).

46 M. Burbano, D. O. Scanlon, and G. W. Watson, J. Am. Chem. Soc. 113, 15065 (2011).

47 D. O. Scanlon, and G. W. Watson, Phys. Rev. Lett. 106, 186403 (2011).

48 J. P. Allen, M. K. Nilsson, D. O. Scanlon, and G. W. Watson, Phys. Rev. B. 83, 035207 (2011).

49 Gaoyang Gou, Ilya Grinberg, Andrew M. Rappe, and James M. Rondinelli, Phys. Rev. B 84, 144101 (2011).

${ }^{50}$ R. Shaltaf, G.-M. Rignanese, X. Gonze, Feliciano Giustino, and Alfredo Pasquarello, Phys. Rev. Lett. 100, 186401 (2008).

${ }^{51}$ Manish Jain, James R. Chelikowsky, and Steven G. Louie, Phys. Rev. Lett. 107, 216806 (2011).

52 M. Ernzerhof and G. E. Scuseria, J. Chem. Phys. 110, 5029 (1999).

53 C. Adamo and V. Barone, J. Chem. Phys. 110, 6158 (1999).

54 J. Heyd, G. E. Scuseria, and M. Ernzerhof, J. Chem. Phys. 124, 219906 (2006)

55 T. M. Henderson, J. Paier, and G. E. Scuseria, Phys. Status Solidi B 248, 767774 (2011).

${ }^{56}$ K. Hummer, J. Harl and G. Kresse, Phys. Rev. B 80, 115205 (2009).

57 J. Wrobel K. J. Kurzydlowski, K. Hummer, G. Kresse, and J. Piechota, Phys. Rev. B 80, 155124 (2009).

58 C. Franchini, A. Sanna, M. Marsman, G. Kresse, Phys. Rev. B 81085213 (2010).

59 R. A. Evarestov, E. Blokhin, D. Gryaznov, E. A. Kotomin, and J. Maier, Phys. Rev. B 83, 134108 (2011).

${ }^{60}$ R. Wahl, D. Vogtenhuber, and G. Kresse, Phys. Rev. B 78, 104116 (2008)

61 T. Archer, C. D. Pemmaraju, S. Sanvito, C. Franchini, J. He, A. Filippetti, P. Delugas, D. Puggioni, V. Fiorentini, R. Tiwari and P. Majumdar, Phys. Rev. B 84, 115114 (2011)

62 C. Franchini, R. Kovacik, M. Marsman, S. Sathyanarayana Murthy, J. He, C. Ederer, G. Kresse, arXiv:1111.1528v1

63 J. M. Rondinelli, Aaron S. Eidelson, and Nicola A. Spaldin, Phys. Rev. B 79, 205119 (2009).

${ }^{64}$ P. Barone, S. Kanungo, S. Picozzi and T. Saha-Dasgupta, Phys. Rev. 84, 134101 (2011).

65 J. B. Goodenough, Phys. Rev. 164, 785 (1967).

66 G. Kresse and J. Hafner, Phys. Rev. B 47, R558 (1993).

67 G. Kresse and J. Furthmuller, Phys. Rev. B 54, 11169 (1996).

68 P.E. Blöchl , Phys. Rev. B 50, 17953 (1994).

${ }^{69}$ G. Kresse and D. Joubert, Phys. Rev. B 59, 1758 (1999).

70 J. Hlinka, J. Petzelt, S. Kamba, D. Noujni, and T. Ostapchuk, Phase Transitions: A Multinational Journal 79, 41 (2006).
71 S. L. Dudarev, G. A. Botton, S. Y. Savrasov, C. J. Humphreys and A. P. Sutton, Phys. Rev. B 57, 1505 (1998).

72 J.P. Perdew, A. Ruzsinszky, G.I. Csonka, O.A. Vydrov, G.E. Scuseria, L.A. Constantin, X. Zhou, and K. Burke, Phys. Rev. Lett. 100, 136406 (2008).

73 In the materials considered here, the equilibrium volumes obtained from HSE and PBEsol $+U$ are essentially the same (see Tables 1 and $\mathrm{V}$ ); hence, the determined U's are essentially independent from our imposing the HSE-volume in the PBEsol $+U$ calculations or not. However, in the general case this may not be true, and it seems reasonable to perform structural relaxations at the DFT $+U$ level for each $U$ considered.

${ }^{74}$ We found two $U$ values, $U=2.2$ and $5.1 \mathrm{eV}$, that generate the same $\Delta E^{+U}=\Delta E^{\mathrm{HSE}}$ for $\mathrm{LaFeO}_{3}$. We chose $5.1 \mathrm{eV}$ because the band gap and magnetic moment are in better agreement with HSE calculations. We also found the FM energy to cross below the AFM one for $U<0.8 \mathrm{eV}$.

75 In our case it takes about 6 days to obtain the phonon frequency for G-AFM BaMnO 3 using a direct HSE calculation with 96 cores (AMD Opteron $61362.4 \mathrm{GHz}$ with Infiniband network), but less then 0.5 hours using PBEsol+U with only 16 cores.

76 Ph.Ghosez, J.-P.Michenaud, and X.Gonze, Phys. Rev. B 58, 6224 (1998)

77 A. Sacchetti, M. Baldini, F. Crispoldi, P. Postorino, P. Dore, A. Nucara, C. Martin, and A. Maignan, Phys. Rev. B 72, 172407 (2005).

78 R. D. Shannon Acta Cryst. A32 751-767 (1976).

79 T. Tohei, A. Kuwabara, T. Yamamoto, F. Oba, and I. Tanaka, Phys. Rev. Lett. 94, 035502 (2005).

80 R. J. Angel, J. Zhao, and N. L. Ross, Phys. Rev. Lett. 95, 025503 (2005).

81 J. Íñiguez and T. Yildirim, Phys. Rev. B 71, 180415 (2005).

82 B.H. Kim, H. Choi, and B.I. Min, New J. Phys. 12, 073023 (2010).

83 Because the symmetry is lower than for a $\operatorname{Pr} \overline{3} m$ perovskite, some modes of the double perovskite $\mathrm{La}_{2} \mathrm{CrFeO}_{6}$ listed in TableVIII are combinations of two modes. We list the dominanant contribution in the Table. For example, the mode with frequency $383 \mathrm{~cm}^{-1}$ in the AFM structure is $86.5 \% \mathrm{R}_{5}^{+}$and $13.5 \% \Gamma_{4}^{-}$, so we denote it as $\mathrm{R}_{5}^{+}$.

84 B.L. Chamberland, A.W. Sleight, and J.F. Weiher, J. Solid State Chem. 1, 506 (1970).

${ }^{85}$ H. Sakai, J. Fujioka, T. Fukuda, D. Okuyama, D. Hashizume, F. Kagawa, H. Nakao, Y. Murakami, T. Arima, A. Q. R. Baron, Y. Taguchi, and Y. Tokura, Phys. Rev. Lett. 107, 137601 (2011).

86 W. E. Pickett, Phys. Rev. B 57, 10613 (1998).

87 K. Miura and K. Terakura, Phys. Rev. B 63, 104402 (2001).

88 K. Ueda, H. Tabata, and T. Kawai, Science 280, 1064 (1998).

89 S. Chakraverty, A. Ohtomo, D. Okuyama, M. Saito, M. Okude, R. Kumai, T. Arima, Y. Tokura, S. Tsukimoto, Y. Ikuhara, and M. Kawasaki, Phys. Rev. B 84, 064436 (2011).

90 L. Schimka, J. Harl, and G. Kresse, J. Chem. Phys. 134, 024116 (2011). 